\title{
DECODING SATELLITE IMAGES OF EARTHQUAKES, PART-III: HARNESSING THE REMOTE SENSING INTELLECTUAL CAPITAL
}

\section{Saumik Dana ${ }^{1, *}$}

${ }^{1}$ University of Southern California, Los Angeles, CA 90089

*Address all correspondence to: Saumik Dana, E-mail: sdana@usc.edu

This document is part III of a series of documents providing a lowdown on image processing in the context of understanding satellite images of earthquakes.

KEY WORDS: Earthquakes, satellite images, remote sensing, image processing

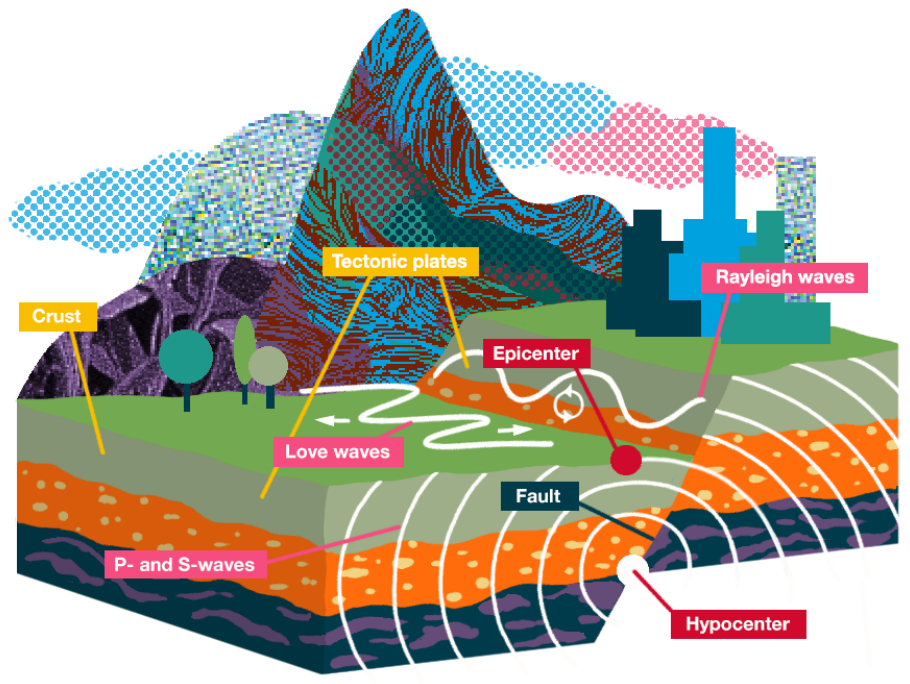

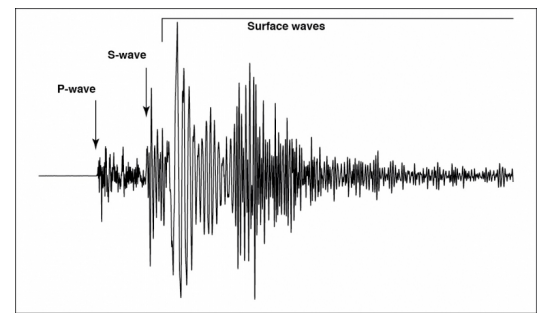

(b) P-, S- and surface waves

(a) Propagation of waves

FIG. 1: Source: Caltech Science Exchange

The earthquake cycle, from slow deformation associated with interseismic behavior to rapid deformation associated with earthquake rupture, spans spatial scales ranging from fractions of a meter associated with the size of contact asperities on faults and individual grains to hundreds of kilometers associated with plate boundaries (Kanamori and Brodsky (2004)). Similarly, temporal scales range from fractions of a second associated with slip at a point during earthquake rupture to hundreds of years of strain accumulation between earthquakes. In many cases, earthquakes are triggered after pore pressure perturbations activate critically stressed seismogenic faults (Kanamori and Brodsky (2004)), not just due to natural causes like earth tides (Scholz et al. (2019)), rainfall (Hainzl et al. (2006)), snowfall (Montgomery-Brown et al. (2019)), typhoons (Liu et al. (2009)), but also due to human activity (Foulger et al. (2018)). As faults slip, the induced stress field spawns seismic waves as shown in Fig. 1a, which travel through and around the 


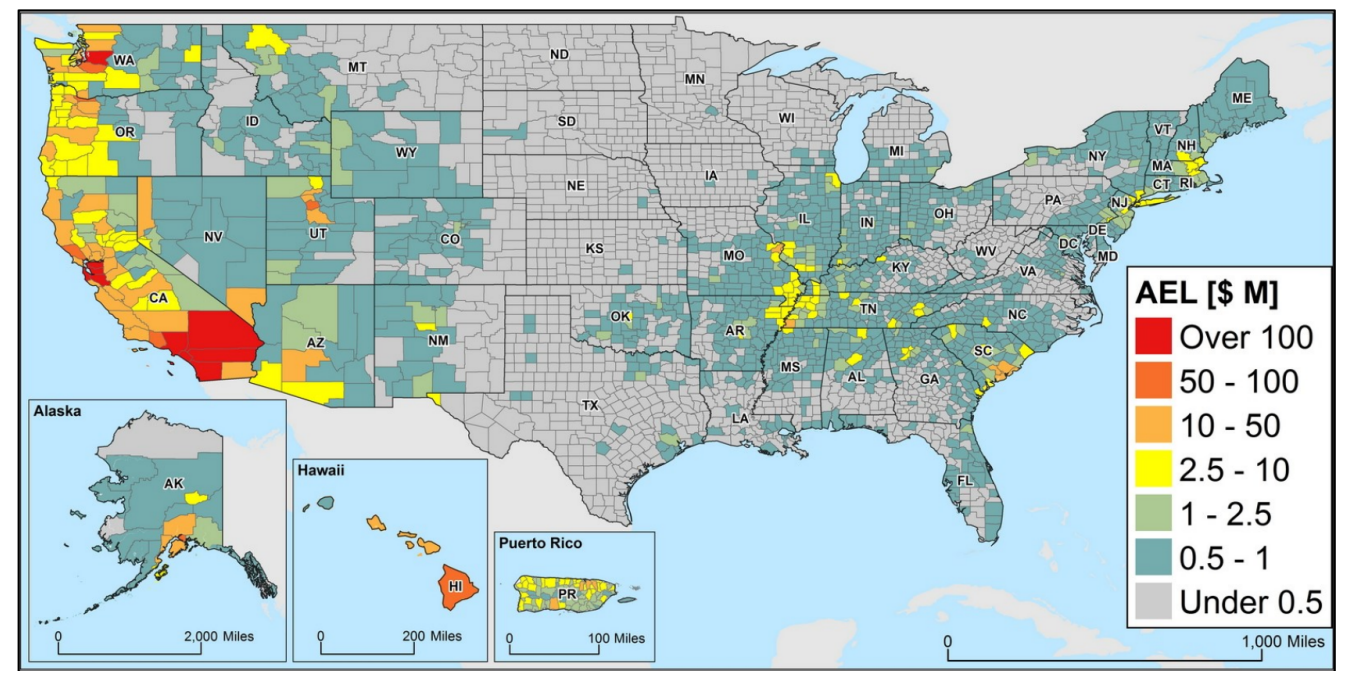

FIG. 2: Annualized Earthquake Losses (AEL: estimated long-term value of earthquake losses to the general building stock) by County. Sources: (1) https://www.usgs.gov/news/usgs-collaborates-fema-national-earthquake-loss-estimate and (2) https://www.fema.gov/hazus. The analysis yielded an estimate of the national AEL of $\$ 6.1$ billion per year. Loss estimates based on the best science and engineering available (during 2016-2017). The study only addresses direct economic losses to buildings, and does not cover damage and losses to critical facilities, transportation and utility lifelines or indirect economic losses.

earth and can be recorded with seismometers as shown in Fig. 1b. Ground motion and failure due to earthquakes lead to huge losses to the tune of billions of dollars annually in the United States (see Fig. 2). Understanding the causality between the events leading to fault slip, the location of the slipping fault and the earthquake recording is important for seismic design and monitoring of underground structures (Hashash et al. (2001)), bridges (Priestley et al. (1996)) and reinforced concrete buildings (Moehle (2015)) as well as climate mitigation projects like carbon sequestration (Zoback and Gorelick (2012)) and energy technologies like enhanced geothermal systems (Ellsworth et al. (2019)) or oilfield wastewater disposal (Keranen and Weingarten (2018)).

Most earthquake detection methods are designed for moderate and large earthquakes. As a consequence, they tend to miss many of the low-magnitude earthquakes that are masked by seismic noise. Detecting and cataloging these earthquakes are key to understanding their causes (natural or human-induced) and, ultimately, to mitigating the seismic risk. Although template matching techniques (which leverage the bank of seismic fingerprints to characterize the seismogram output) have seen a lot of advances in the past decade (Yoon et al. (2015)), they still suffer from poor earthquake location estimates. On the other hand, harnessing the remote sensing intellectual capital offers a lot of avenues for earthquake location estimates. According to USGS, remote sensing is the process of detecting and monitoring the physical characteristics of an area by measuring its reflected and emitted radiation at a distance (typically from satellite or aircraft). Special cameras collect remotely sensed images, which help researchers "sense" things about the Earth. Some examples are:

$\checkmark$ Cameras on satellites and airplanes take images of large areas on the Earth's surface, allowing us to see much more than we can see when standing on the ground.

$\checkmark$ Sonar systems on ships can be used to create images of the ocean floor without needing to travel to the bottom of the ocean.

$\checkmark$ Cameras on satellites can be used to make images of temperature changes in the oceans.

A holistic framework to study earthquakes would incorporate seismic wave propagation and subsurface pressure perturbations, and have a dialogue with the remote sensing based inverse modeling framework. The first step is working quasi-static simulations (Dana (2018, 2019a,b); Dana et al. (2018, 2020a); Dana and Jha (2021); Dana and Lyathakula (2021); Dana et al. (2020b); Dana and Wheeler (2018a,b,c, 2019, 2020a,b); Dana et al. (2021)) in which wave propagation is ignored and the solution is a time series of solutions to static problems with potentially 
time-varying physical properties and boundary conditions. The next step is to gradually leverage this subject matter expertise to render fully dynamic simulations. The next step is to arrive at deployable remote sensing techniques as an inverse modeling framework to work with the aforementioned forward modeling framework.

\subsection{Image processing in remote sensing}

The image processing techniques typically used in remote sensing are:

$\checkmark$ Image enhancement

$\checkmark$ Feature extraction

$\checkmark$ Segmentation

$\checkmark$ Image classification

$\checkmark$ Feature detection

\subsubsection{Image enhancement}

Contrast refers to the amount of differentiation that is there between the various image features. Images having a higher contrast level generally display a greater degree of color or gray-scale variation than those of lower contrast.

$\checkmark$ Histogram equalization is an image processing technique that adjusts image intensities to improve contrast. Histogram Equalization is one of the simplest and commonly used method in low level image enhancement using the histogram. The logic behind Histogram Equalization is that the image with the best visual appearance, is the one whose histogram looks like the regular distribution.

$\checkmark$ Adaptive Histogram Equalization computes many histograms for each of the separate part of the image, and uses them to redistribute the lightness values of the image, hence it differs from Histogram Equalization. Hence it is suitable for bettering the local contrast in images.

$\checkmark$ In Contrast Stretching the contrast in an image is stretched from the range of intensity values it contains to span a desired range of values. It is also called Normalization. Some Contrast Stretching techniques include: Minimum-Maximum, Percentage, and Piecewise Contrast Enhancement.

\section{1 .2 Feature extraction}

Machines store images as a collection of numbers between 0 and 255 called pixels. Any image can be represented as a combination of three colors, red, green, and blue. In essence, any image can represented as a collection of pixels in three channels, with each channel representing either red, green, or blue. To interpret this, let us say that a pixel in an image is a collection of a tuple like $(34,243,8)$, which means that the pixel is " $34 / 256$ in redness", "244/256 in greenness", and "9/256 in blueness". One of the ways to extract features is to take the mean value for each pixel i.e $(34+244+8) / 3$ for this particular pixel, and create a feature vector. The number of entries in the vector is the number of pixels in the image. One more feature extraction method is edge detection, which looks for sharp changes in pixel values. To automate the process, there are various kernels which are used to operate on each pixel to "manufacture a neighborhood around each pixel", which then exposes the edge pixels.

\subsubsection{Image segmentation}

Segmentation is about clustering a bunch of pixels into a common group.

$\checkmark$ Region-Based Segmentation: Separates the objects into different regions based on some threshold value(s)

$\checkmark$ Edge Detection Segmentation: Makes use of discontinuous local features of an image to detect edges and hence define a boundary of the object.

$\checkmark$ Segmentation based on Clustering: Divides the pixels of the image into homogeneous clusters.

$\checkmark$ Mask R-CNN: Gives three outputs for each object in the image: its class, bounding box coordinates, and object mask 


\subsubsection{Image classification}

Image classification is a method of pattern recognition in which images/pixels are classified based on some similarity measures

$\checkmark$ Supervised method needs the training data to teach the classifier and recognize the instances of necessary details in the image. It is divided into parametric and non-parametric methods where parametric method use algebraic method for assignment to classes. Bayesian and decision tree come under this category. Non-parametric method is used when density function is not present. K-Nearest Neighbor and Logical Regression come under this category.

$\checkmark$ Unsupervised method finds group of similar instances with no information about class label. Unsupervised methods can be hierarchical and partition clustering

\subsubsection{Feature detection}

$\checkmark$ Harris corner detection: As shown in Fig. 3, corners are detected using the idea that shifting the window in any direction should yield a large change in appearance. The algorithm is given in Algorithm 1.

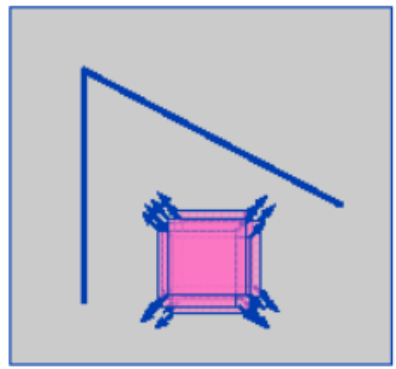

"flat" region: no change in all directions

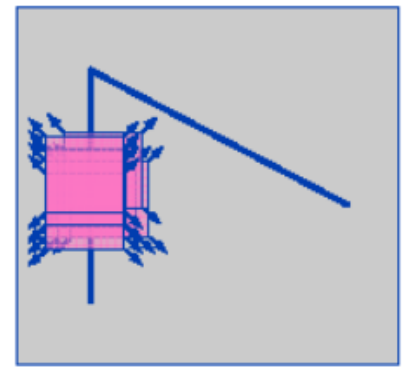

"edge":

no change along

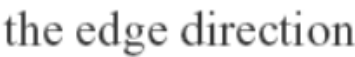

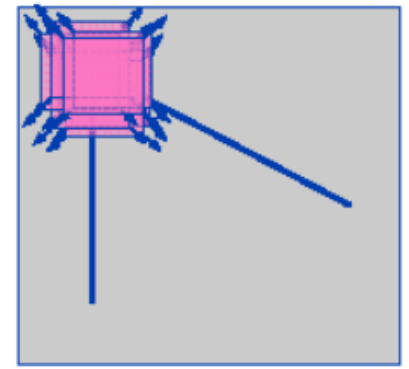

"corner": significant change in all directions

FIG. 3: Harris corner detector gives a mathematical approach for determining which case holds

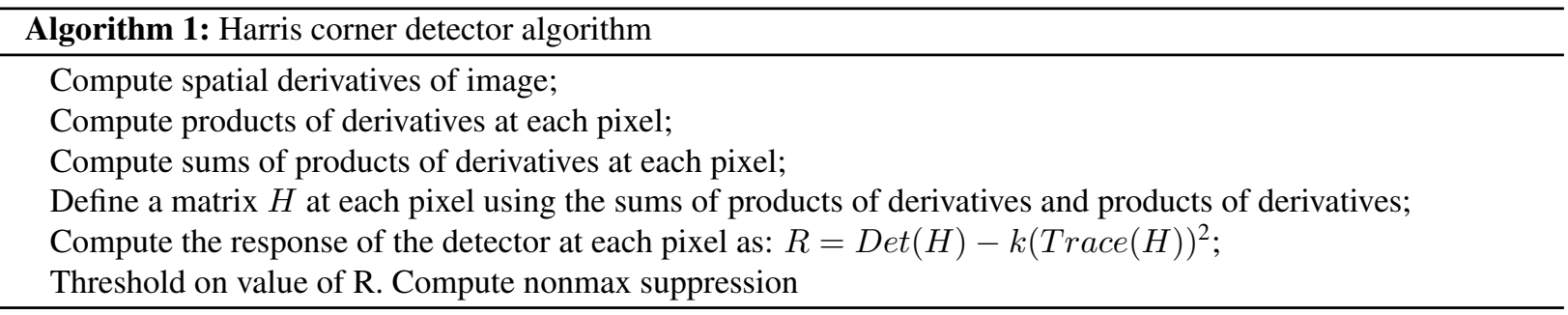

$\checkmark$ Shi-Tomasi corner detection: The difference between the Harris corner detector and Shi-Tomasi corner detector is the value of $R$. It is computed as the minimum eigenvalue of matrix $H$ instead of $R=\operatorname{Det}(H)-$ $k(\operatorname{Trace}(H))^{2}$

$\checkmark$ Scale-invariant feature transform: The scale-invariant feature transform (SIFT) is an algorithm used to detect and describe local features in digital images. It locates certain key points and then furnishes them with quantitative information (so-called descriptors) which can for example be used for object recognition. The descriptors are supposed to be invariant against various transformations which might make images look different although they represent the same object(s). The steps are: 
- Constructing a scale space: This is the initial preparation. You create internal representations of the original image to ensure scale invariance. This is done by generating a "scale space".

- LoG Approximation: The Laplacian of Gaussian is great for finding interesting points (or key points) in an image. But it's computationally expensive. So we cheat and approximate it using the representation created earlier.

- Finding keypoints: With the super fast approximation, we now try to find key points. These are maxima and minima in the Difference of Gaussian image we calculate in step 2

- Get rid of bad key points: Edges and low contrast regions are bad keypoints. Eliminating these makes the algorithm efficient and robust. A technique similar to the Harris Corner Detector is used here.

- Assigning an orientation to the keypoints: An orientation is calculated for each key point. Any further calculations are done relative to this orientation. This effectively cancels out the effect of orientation, making it rotation invariant.

- Generate SIFT features: Finally, with scale and rotation invariance in place, one more representation is generated. This helps uniquely identify features.

$\checkmark$ Speeded-up robust features (SURF): SURF is an improvement over SIFT. In SIFT, images are repeatedly smoothed with a Gaussian and subsequently sub- sampled. Alternatively, in SURF, filters of larger size on the original image are used. Due to using integral images, filters of any size can be applied at exactly the same speed. The "integral image" of an image represents the sum of all pixels in the image of a rectangular region formed by $(0,0)$ and $(x, y)$. Furthermore, instead of using a different measure for selecting the location and scale of interest points (e.g., Hessian and difference of Gaussian in SIFT), SURF uses the determinant of the constructed modified Hessian to find both.

\section{References}

Dana, S., Addressing challenges in modeling of coupled flow and poromechanics in deep subsurface reservoirs, $\mathrm{PhD}$ thesis, The University of Texas at Austin, 2018.

Dana, S., A simple framework for arriving at bounds on effective moduli in heterogeneous anisotropic poroelastic solids, arXiv preprint arXiv:1912.10835, 2019a.

Dana, S., System of equations and staggered solution algorithm for immiscible two-phase flow coupled with linear poromechanics, arXiv preprint arXiv:1912.04703, 2019b.

Dana, S., Ganis, B., and Wheeler, M.F., A multiscale fixed stress split iterative scheme for coupled flow and poromechanics in deep subsurface reservoirs, Journal of Computational Physics, vol. 352, pp. 1-22, 2018.

Dana, S., Ita, J., and Wheeler, M.F., The correspondence between voigt and reuss bounds and the decoupling constraint in a twogrid staggered algorithm for consolidation in heterogeneous porous media, Multiscale Modeling \& Simulation, vol. 18, no. 1, pp. 221-239, 2020a.

Dana, S. and Jha, B., A fault slip model to study earthquakes due to pore pressure perturbations, arXiv preprint arXiv:2104.06257, 2021.

Dana, S. and Lyathakula, K.R., Uncertainty quantification in friction model for earthquakes using bayesian inference, arXiv preprint arXiv:2104.11156, 2021.

Dana, S., Srinivasan, S., Karra, S., Makedonska, N., Hyman, J.D., O’Malley, D., Viswanathan, H., and Srinivasan, G., Towards real-time forecasting of natural gas production by harnessing graph theory for stochastic discrete fracture networks, Journal of Petroleum Science and Engineering, vol. 195, p. 107791, $2020 \mathrm{~b}$.

Dana, S. and Wheeler, M.F., Augmented lagrangian for treatment of hanging nodes in hexahedral meshes, arXiv preprint arXiv:1809.04031, 2018a.

Dana, S. and Wheeler, M.F., Convergence analysis of fixed stress split iterative scheme for anisotropic poroelasticity with tensor biot parameter, Computational Geosciences, vol. 22, no. 5, pp. 1219-1230, $2018 \mathrm{~b}$. 
Dana, S. and Wheeler, M.F., Convergence analysis of two-grid fixed stress split iterative scheme for coupled flow and deformation in heterogeneous poroelastic media, Computer Methods in Applied Mechanics and Engineering, vol. 341, pp. 788-806, 2018c.

Dana, S. and Wheeler, M.F., Design of convergence criterion for fixed stress split iterative scheme for small strain anisotropic poroelastoplasticity coupled with single phase flow, arXiv preprint arXiv:1912.06476, 2019.

Dana, S. and Wheeler, M.F., An efficient algorithm for numerical homogenization of fluid filled porous solids: part-i, arXiv preprint arXiv:2002.03770, 2020a.

Dana, S. and Wheeler, M.F., A machine learning accelerated $\mathrm{fe}^{2}$ homogenization algorithm for elastic solids, arXiv preprint arXiv:2003.11372, 2020b.

Dana, S., Zhao, X., and Jha, B., Two-grid method on unstructured tetrahedra: Applying computational geometry to staggered solution of coupled flow and mechanics problems, arXiv preprint arXiv:2102.04455, 2021.

Ellsworth, W.L., Giardini, D., Townend, J., Ge, S., and Shimamoto, T., Triggering of the pohang, korea, earthquake (m w 5.5) by enhanced geothermal system stimulation, Seismological Research Letters, vol. 90, no. 5, pp. 1844-1858, 2019.

Foulger, G.R., Wilson, M.P., Gluyas, J.G., Julian, B.R., and Davies, R.J., Global review of human-induced earthquakes, EarthScience Reviews, vol. 178, pp. 438-514, 2018.

Hainzl, S., Kraft, T., Wassermann, J., Igel, H., and Schmedes, E., Evidence for rainfall-triggered earthquake activity, Geophysical Research Letters, vol. 33, no. 19, 2006.

Hashash, Y.M., Hook, J.J., Schmidt, B., John, I., and Yao, C., Seismic design and analysis of underground structures, Tunnelling and underground space technology, vol. 16, no. 4, pp. 247-293, 2001.

Kanamori, H. and Brodsky, E.E., The physics of earthquakes, Reports on Progress in Physics, vol. 67, no. 8, p. $1429,2004$.

Keranen, K.M. and Weingarten, M., Induced seismicity, Annual Review of Earth and Planetary Sciences, vol. 46, pp. 149-174, 2018.

Liu, C., Linde, A.T., and Sacks, I.S., Slow earthquakes triggered by typhoons, Nature, vol. 459, no. 7248, pp. 833-836, 2009.

Moehle, J., Seismic design of reinforced concrete buildings, McGraw-Hill Education, 2015.

Montgomery-Brown, E., Shelly, D.R., and Hsieh, P.A., Snowmelt-triggered earthquake swarms at the margin of long valley caldera, california, Geophysical Research Letters, vol. 46, no. 7, pp. 3698-3705, 2019.

Priestley, M.N., Seible, F., and Calvi, G.M., Seismic design and retrofit of bridges, John Wiley \& Sons, 1996.

Scholz, C.H., Tan, Y.J., and Albino, F., The mechanism of tidal triggering of earthquakes at mid-ocean ridges, Nature communications, vol. 10, no. 1, pp. 1-7, 2019.

Yoon, C.E., O’Reilly, O., Bergen, K.J., and Beroza, G.C., Earthquake detection through computationally efficient similarity search, Science advances, vol. 1, no. 11, p. e1501057, 2015.

Zoback, M.D. and Gorelick, S.M., Earthquake triggering and large-scale geologic storage of carbon dioxide, Proceedings of the National Academy of Sciences, vol. 109, no. 26, pp. 10164-10168, 2012. 